\title{
Anti-Hyperglycemic Effect of a Kudzu (Pueraria lobata) Vine Extract in Ovariectomized Mice
}

\author{
Teruyoshi TANAKA, Yukihiro YoKOTA, Hanjun TANG, Nobuhiro Zaima, \\ Tatsuya MORIYAMA and Yukio KAWAMURA* \\ Department of Applied Biological Chemistry, Graduate School of Agriculture, \\ Kinki University, 3327-204, Naka-machi, Nara 631-8505, Japan
}

(Received November 2, 2015)

\begin{abstract}
Summary Postmenopausal diabetes is exacerbated by estrogen deficiency. Ovariectomized (OVX) animal models can be used to develop strategies for preventing or treating postmenopausal symptoms. We previously found that a diet containing kudzu (Pueraria lobata) vine ethanol extract (PVEE) suppressed weight gain in OVX mice. Therefore, this study further elucidated how PVEE affected OVX mice. Ten-week-old OVX or sham-operated mice were fed diets containing either no PVEE (control) or $20 \mathrm{mg} \cdot \mathrm{kg}^{-1} \cdot \mathrm{d}^{-1}$ PVEE for $8 \mathrm{wk}, 5 \mathrm{mg} \cdot \mathrm{kg}^{-1} \cdot \mathrm{d}^{-1}$ PVEE for $24 \mathrm{wk}$, or $20 \mathrm{mg} \cdot \mathrm{kg}^{-1} \cdot \mathrm{d}^{-1}$ puerarin (daidzein-8-C-glucoside), a major isoflavone present in PVEE, for $10 \mathrm{wk}$. The effects of puerarin on glucose tolerance were also tested in OVX mice. The experimental diets were not associated with any abnormalities in any mice tested in the present study. Weight gain and serum glucose levels were increased in OVX mice and these effects were significantly attenuated in OVX mice that consumed PVEE ( 5 or $20 \mathrm{mg} \cdot \mathrm{kg}^{-1} \cdot \mathrm{d}^{-1}$ ) or puerarin. Puerarin-treated OVX mice also showed reduced serum glucose levels following administration of $1,000 \mathrm{mg} \cdot \mathrm{kg}^{-1}$ glucose. These results suggested that puerarin contributed to PVEE-mediated improvements in glucose metabolism in OVX mice. Although further studies are needed to clarify the molecular mechanism underlying these observations, PVEE and puerarin could provide effective approaches to the amelioration of postmenopausal diabetes.
\end{abstract}

Key Words Kudzu, Pueraria lobata, postmenopausal diabetes, puerarin, glucose metabolism

Postmenopausal women have an increased risk for metabolic diseases such as obesity and diabetes $(1,2)$, which increase their risk of developing other serious conditions including cancers, neurological disorders, hypertension, and other kinds of cardiovascular disease (3). Postmenopausal diabetes is induced by estrogen deficiency, which exacerbates insulin resistance $(4,5)$. The number of postmenopausal patients with diabetes has recently increased, due to the increasing age of the population. Therefore, dietary strategies to reduce the risk for diabetes are expected to be necessary in order to improve postmenopausal health.

Kudzu (Pueraria lobata) is a creeping vine of the Leguminosae family. The plant has spread worldwide and is predominantly found in temperate climates. Dried kudzu roots (Puerariae radix) are traditionally used in Asian countries, including Japan, as an herbal medicine to treat the common cold, headaches, diarrhea, and hypertension. Prasain et al. demonstrated that kudzu root extract ameliorated impaired glucose and lipid metabolism in obese male mice (6). They also reported that in addition to lowering arterial blood pressure, a

\footnotetext{
${ }^{*}$ To whom correspondence should be addressed at present address: Department of Food Nutrition and Biochemistry, Kyoto Women's University, Kitahiyoshi-cho 35, Imakumano, Higashiyama-ku, Kyoto 605-8501, Japan

E-mail: kawamury@kyoto-wu.ac.jp
}

kudzu root extract diet significantly reduced blood cholesterol, glucose, and insulin levels in stroke-prone spontaneously hypertensive rats (7).

Pharmaceutical legislation in Japan classifies the kudzu root as a medicinal resource, rather than a food, and we therefore investigated whether dietary consumption of other parts of the plant had beneficial effects on postmenopausal diabetes. Our previous study of a kudzu vine ethanol extract (PVEE) showed that dietary PVEE decreased body weight in ovariectomized (OVX) mice (8). Accordingly, more research is required to elucidate how PVEE affects estrogen deficiency.

OVX animals are used as a model of postmenopausal diabetes (9-11). OVX mice show glucose intolerance, insulin resistance, and increased body weight $(10,11)$. This study investigated whether and how dietary administration of PVEE improved glucose metabolism in OVX mice.

\section{MATERIALS AND METHODS}

Materials. PVEE was prepared as described previously (8). Briefly, 3-5 volumes of ethanol were added to kudzu vines per unit weight, and the mixture was subsequently homogenized. After filtration, the extracts were concentrated in an evaporator. Finally, the extracts were frozen in liquid nitrogen and dried.

Puerarin $(96 \%$ purity) was purchased from LKT Laboratories (St. Paul, MN) and $17 \beta$-estradiol (E2) was 
purchased from Wako Pure Chemical Industries, Ltd. (Osaka, Japan). All other chemicals used in this study were of the highest purity available.

Animals and administration. All animal experiments were approved by the Institutional Animal Care and Use Committee and carried out according to the Kinki University Animal Experimentation Regulations (approval ID: KAAG-19-003).

Experiment 1: Slc:ddY female mice were purchased from Japan SLC, Inc. (Shizuoka, Japan). Mice were either OVX or sham-operated (Sham) at $9 \mathrm{wk}$ of age. Sham and OVX mice were divided into Sham-control $(n=6)$, Sham-PVEE $(n=6)$, OVX-control $(n=6)$, and OVX-PVEE groups $(n=6)$. Commercial diet (MF; Oriental Yeast Co., Ltd., Tokyo, Japan) was used as the control diet. A PVEE diet $\left(20 \mathrm{mg} \cdot \mathrm{kg}^{-1} \cdot \mathrm{d}^{-1}\right.$, delivering approximately $0.66 \mathrm{mg} \mathrm{PVEE} \cdot \mathrm{d}^{-1} \cdot$ animal $^{-1}$ ) was prepared by adding PVEE to MF powder. This dosage was calculated on the basis of the isoflavonoid level recommended as a Food for Specified Health Uses in Japan, whereby 30-40 mg supplementation $\cdot \mathrm{d}^{-1}$ for a $60-\mathrm{kg}$ man corresponded to $0.5-0.66 \mathrm{mg} \cdot \mathrm{d}^{-1}$. for a mouse. A very small amount of PVEE was added to the MF diet and no cornstarch was added to the control diet to compensate for this. The mice were housed individually under a 12-h/12-h light/ dark cycle at $20 \pm 2^{\circ} \mathrm{C}$. Diet and tap water were supplied ad libitum. The mice were sacrificed under anesthesia after 8 wk of PVEE or control diet intake, and the uteri, white adipose tissue in the epoophoron, and blood samples were collected. The relative weights of the uteri and white adipose tissue (against body weight $\times 100$ ) were calculated.

Experiment 2: Thirteen-week-old OVX or sham-operated mice were divided into Sham-control $(n=6)$, ShamPVEE $(n=6)$, OVX-control $(n=6)$, and OVX-PVEE groups $(n=6)$. A PVEE diet $\left(5 \mathrm{mg} \cdot \mathrm{kg}^{-1} \cdot \mathrm{d}^{-1}\right)$ was prepared by adding PVEE to MF powder. Sham and OVX mice were fed the control or PVEE diet for 24 wk. Other experimental conditions were identical to those described in Experiment 1.

Experiment 3: Nine-week-old OVX or sham-operated mice were divided into Sham-control $(n=8)$, OVX-control $(n=8)$, OVX-puerarin $(n=10)$, and OVX-E2 groups $(n=8)$. A puerarin diet $\left(20 \mathrm{mg} \cdot \mathrm{kg}^{-1} \cdot \mathrm{d}^{-1}\right)$ was prepared by adding puerarin to MF powder. Sham and OVX mice were fed the control or puerarin diet for $10 \mathrm{wk}$. The mice in the OVX-E2 group were intraperitoneally injected with a daily dose of $40 \mu \mathrm{g} \cdot \mathrm{kg}^{-1} \mathrm{E} 2$. The mice in the other groups were injected with vehicle daily. Other experimental conditions were identical to those described in Experiment 1.

Experiment 4: Nine-week-old OVX mice were divided into the OVX-control $(n=5)$ and OVX-puerarin groups $(n=5)$. Nine-week-old Sham mice were also divided into the Sham-control $(n=6)$ and Sham-puerarin groups $(n=6)$. The mice were deprived of food for $18 \mathrm{~h}$ prior to testing their glucose tolerance by oral administration of glucose $\left(1,000 \mathrm{mg} \cdot \mathrm{kg}^{-1}\right)$, with or without puerarin $\left(50 \mathrm{mg} \cdot \mathrm{kg}^{-1}\right)$. Blood was collected from the tail vein 0-120 min after the administration of glucose.
Computed tomography. The mouse bodies were scanned using an X-ray computed tomography system (LCT-100; ALOKA, Tokyo, Japan). Tomograms were taken at $0.5-\mathrm{mm}$ intervals from the diaphragm to the bottom of the abdominal cavity. Fat mass (total, visceral, and subcutaneous fat) was measured by analyzing the tomograms. The data were presented as the relative fat mass (against body weight $\times 100$ ).

Triglyceride, cholesterol, and glucose levels. Serum glucose, triglyceride, total cholesterol, and high-density lipoprotein (HDL) cholesterol levels were determined using triglyceride-E, cholesterol E, and HDL-C kits (Wako Pure Chemical Industries, Ltd.), respectively. Serum lowdensity lipoprotein (LDL) cholesterol levels were calculated from the total cholesterol, HDL-cholesterol, and triglyceride levels.

Liver lipids were extracted according to the method of Bligh and Dyer (12) and evaporated under $\mathrm{N}_{2}$. The extract was dissolved in isopropanol prior to measurement of the liver triglyceride and cholesterol levels using the above-mentioned kits.

Serum insulin, leptin, and adiponectin levels. Serum insulin levels were measured using an enzyme-linked immunosorbent assay kit (Morinaga Institute of Biological Science, Kanagawa, Japan). Serum leptin levels were measured using a rat leptin enzyme-linked immunosorbent assay kit (Morinaga Institute of Biological Science). Serum adiponectin levels were determined using a commercial kit (Acrp30; R\&D Systems, Minneapolis, MN).

Statistical analysis. Data are presented as the mean \pm standard error of the mean. One-way analysis of variance followed by a post-hoc Tukey test was used to examine differences between groups. The level of significance was set at $p<0.05$.

\section{RESULTS}

\section{Effects of the PVEE diet on body and uterine weight}

In this study (experiment 1), a diet containing $20 \mathrm{mg} \cdot \mathrm{kg}^{-1} \cdot \mathrm{d}^{-1}$ PVEE was fed to Sham and OVX mice for $8 \mathrm{wk}$. Figure 1A shows the mouse body weights over the course of the experiment. From 2 wk onwards, the body weight of the OVX-control group (36.1 $00.6 \mathrm{~g})$ was significantly greater than that of the Sham-control group $(33.2 \pm 0.7 \mathrm{~g})$. The PVEE diet significantly suppressed this OVX-induced weight gain from 7 wk onwards. The final body weights in the Sham-control, Sham-PVEE, OVX-control, and OVX-PVEE groups were 36.3 \pm 1.0 , $34.9 \pm 0.8,39.4 \pm 0.2$, and $37.9 \pm 0.4 \mathrm{~g}$, respectively. Food intake did not differ significantly among the 4 groups; the mean food intake in the Sham-control, Sham-PVEE, OVX-control, and OVX-PVEE groups during experiment 1 was $4.6 \pm 0.1,4.4 \pm 0.1,4.5 \pm 0.1$, and $4.5 \pm 0.1 \mathrm{~g}$, respectively. As shown in Fig. 1B, the relative uterine weight (against body weight $\times 100$ ) in OVXcontrol mice was significantly lower than that of the Sham-control mice, suggesting that uterine atrophy had occurred, as a result of estrogen deficiency. Administration of the PVEE diet did not restore this decrease in the relative uterine weight in OVX mice. These results suggested that PVEE suppressed OVX-induced weight gain, 
A

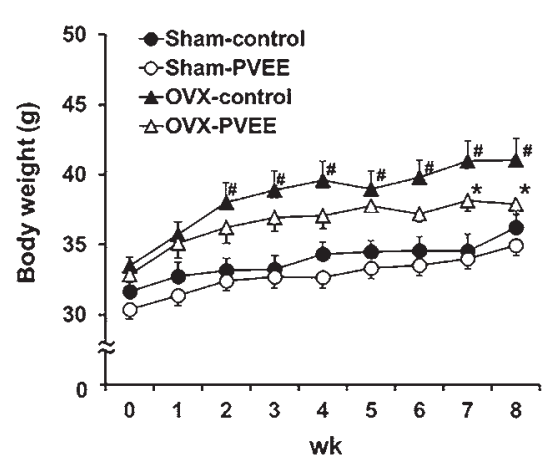

B

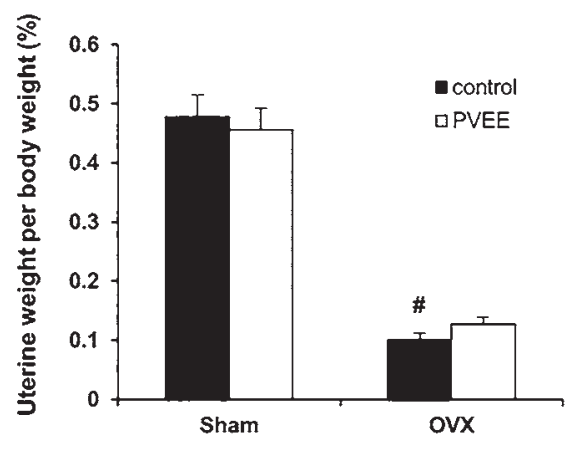

Fig. 1. Effects of the kudzu vine ethanol extract (PVEE) diet on body weight (A) and uterine weight at 8 wk (B) in the shamoperated (Sham)-control group ( $n=6$, closed circles), Sham-PVEE group ( $20 \mathrm{mg} \cdot \mathrm{kg}^{-1} \cdot \mathrm{d}^{-1}, n=6$, open circles), ovariectomized (OVX)-control group ( $n=6$, closed triangles), and OVX-PVEE group $\left(20 \mathrm{mg} \cdot \mathrm{kg}^{-1} \cdot \mathrm{d}^{-1}, n=6\right.$, open triangles). Data are presented as mean \pm standard error. ${ }^{*} p<0.05$ vs. Sham-control group. ${ }^{*} p<0.05$ vs. OVX-control group.

A Total fat mass
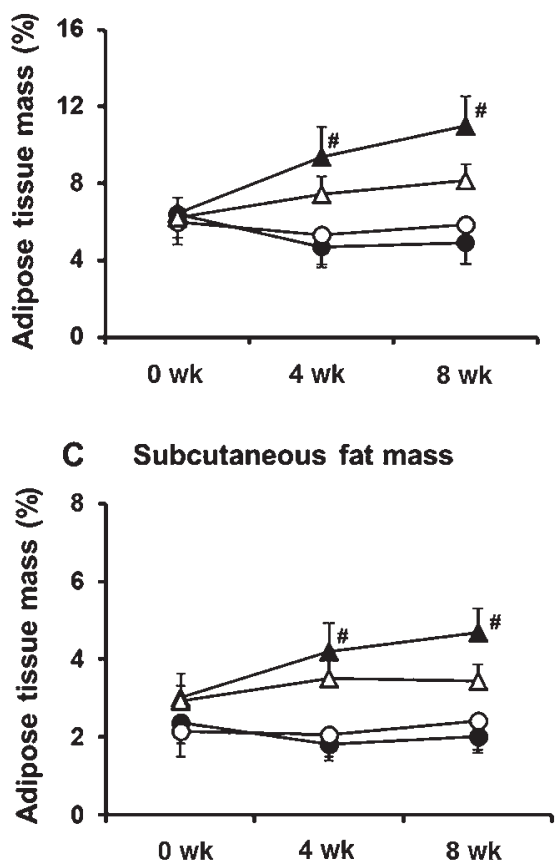

B Visceral fat mass
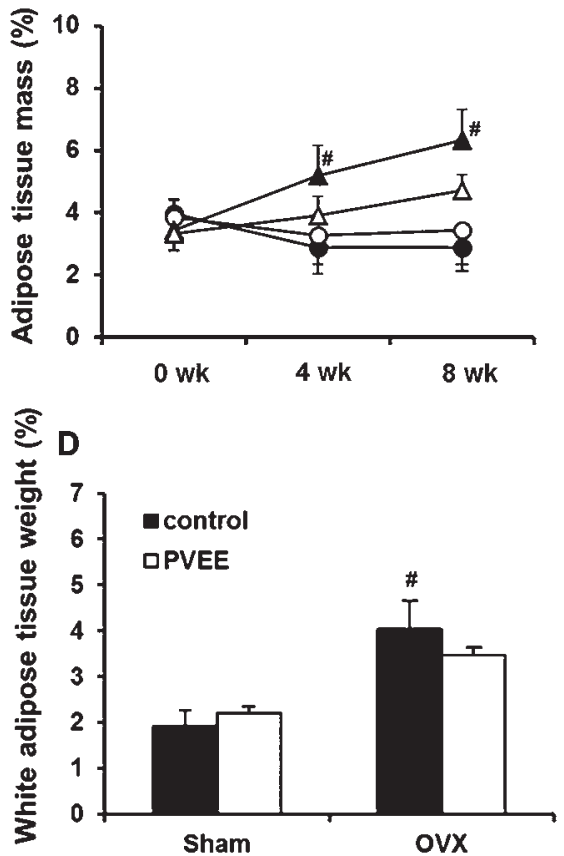

Fig. 2. Total fat mass (A), visceral fat mass (B), and subcutaneous fat mass (C) in the sham-operated (Sham)-control group ( $n=6$, closed circles), Sham-kudzu vine ethanol extract (PVEE) group (20 mg $\mathrm{kg}^{-1} \cdot \mathrm{d}^{-1}, n=6$, open circles), ovariectomized (OVX)-control group ( $n=6$, closed triangles), and OVX-PVEE group (20 $\mathrm{mg} \cdot \mathrm{kg}^{-1} \cdot \mathrm{d}^{-1}, n=6$, open triangles). After consuming the PVEE diet for $8 \mathrm{wk}$, white adipose tissue weight in the epoophoron was measured (D). Data are presented as mean \pm standard error. ${ }^{\#} p<0.05$ vs. Sham-control group.

without altering uterine weight.

Effect of the PVEE diet on adipose tissue mass

$\mathrm{X}$-ray computed tomography analysis was performed to determine the fat mass-body weight ratio during experiment 1 (Fig. 2A-C). The OVX-control group had significantly more total, visceral, and subcutaneous fat than the Sham-control group. Although the mice receiving dietary PVEE showed a reduced increase in fat mass after 4 and $8 \mathrm{wk}$, there were no significant differences between the OVX-control and OVX-PVEE groups. At $8 \mathrm{wk}$, the relative total, visceral, and subcutaneous fat levels in the OVX-PVEE group were $74.3 \%, 74.5 \%$, and $73.7 \%$ of the corresponding values in the OVX-control group, respectively.

As shown in Fig. 2D, the relative white adipose tissue weight (against body weight $\times 100$ ) in the epoophoron of the OVX-control group was higher than that of the Sham-control group. OVX-PVEE mice showed a $19 \%$ lower increase in relative white adipose tissue weight, as compared with the OVX-control group, although this difference was not statistically significant. These findings were concordant with those of the computed tomography analysis. Furthermore, the PVEE diet did not alter the relative lung, heart, liver, spleen, or kidney weights 
Table 1. Effect of $20 \mathrm{mg} \cdot \mathrm{kg}^{-1} \cdot \mathrm{d}^{-1} \mathrm{kudzu}$ vine ethanol extract (PVEE) diet on serum parameters after $8 \mathrm{wk}$.

\begin{tabular}{|c|c|c|c|c|}
\hline & Sham-control & Sham-PVEE & OVX-control & OVX-PVEE \\
\hline Triglyceride (mg/dL) & $58.0 \pm 4.2$ & $53.8 \pm 1.7$ & $68.8 \pm 6.1$ & $60.4 \pm 3.0$ \\
\hline Total cholesterol (mg/dL) & $107.0 \pm 8.3$ & $112.9 \pm 6.8$ & $134.2 \pm 5.4$ & $127.1 \pm 7.1$ \\
\hline HDL-cholesterol (mg/dL) & $50.9 \pm 4.3$ & $55.7 \pm 1.9$ & $65.3 \pm 4.0$ & $64.4 \pm 6.9$ \\
\hline LDL-cholesterol (mg/dL) & $45.8 \pm 2.0$ & $50.7 \pm 1.4$ & $51.0 \pm 4.7$ & $50.6 \pm 3.3$ \\
\hline Glucose $(\mathrm{mg} / \mathrm{dL})$ & $108.7 \pm 6.4$ & $98.4 \pm 3.1$ & $130.4 \pm 2.1^{\mathrm{a}}$ & $94.1 \pm 2.4^{\mathrm{b}}$ \\
\hline Insulin (ng/mL) & $0.6 \pm 0.2$ & $0.7 \pm 0.2$ & $1.2 \pm 0.4$ & $0.9 \pm 0.2$ \\
\hline Leptin (ng/mL) & $1.2 \pm 0.2$ & $1.4 \pm 0.2$ & $2.0 \pm 0.4^{\mathrm{a}}$ & $1.5 \pm 0.2$ \\
\hline Adiponectin $(\mu \mathrm{g} / \mathrm{mL})$ & $4.3 \pm 0.6$ & $3.3 \pm 0.4$ & $3.7 \pm 0.3$ & $2.4 \pm 0.1$ \\
\hline
\end{tabular}

Data are presented as mean \pm standard error; ${ }^{a} p<0.05$ vs. Sham-control group; ${ }^{b} p<0.05$ vs. OVX-control group. Sham, sham-operated; OVX, ovariectomized; HDL, high-density lipoprotein; LDL, low-density lipoprotein.
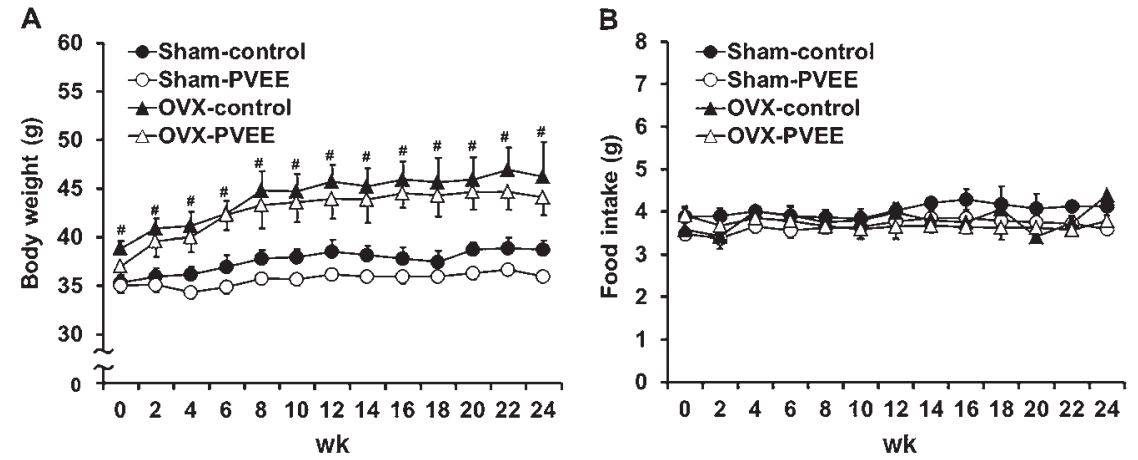

Fig. 3. Effects of long-term intake of the kudzu vine ethanol extract (PVEE) diet on body weight (A) and food intake (B) in the sham-operated (Sham)-control group ( $n=6$, closed circles), Sham-PVEE group $\left(5 \mathrm{mg} \cdot \mathrm{kg}^{-1} \cdot \mathrm{d}^{-1}, n=6\right.$, open circles), ovariectomized (OVX)-control group ( $n=6$, closed triangles), and OVX-PVEE group $\left(5 \mathrm{mg} \cdot \mathrm{kg}^{-1} \cdot \mathrm{d}^{-1}, n=6\right.$, open triangles). Data are presented as mean \pm standard error. ${ }^{\#} p<0.05$ vs. Sham-control group.

in OVX mice (data not shown). These results suggested that the PVEE diet reduced body weight by suppressing fat accumulation.

Effects of the PVEE diet on serum parameters

We investigated whether dietary PVEE affected serum triglyceride, total cholesterol, and HDL- or LDL-cholesterol levels (Table 1). OVX mice showed higher serum triglyceride, cholesterol, and HDL-cholesterol levels, and lower LDL-cholesterol, as compared to the Sham-control group, although these differences were not statistically significant. The serum triglyceride and cholesterol levels in OVX-PVEE group tended to decrease, although these changes were not significant. In addition, the PVEE diet did not alter serum HDL- or LDL-cholesterol levels.

Next, we examined the effects of the PVEE diet on serum glucose and insulin levels. As shown in Table 1, the serum glucose levels in the OVX-control group were significantly higher than those in the Sham-control group. The PVEE diet significantly attenuated this OVXassociated elevation in the serum glucose level. The PVEE diet significantly attenuated this OVX-associated elevation in the serum glucose level. Serum insulin levels were increased in the OVX mice and the PVEE diet attenuated this effect (Table 1), although this difference was not statistically significant. These results suggested that PVEE improves serum glucose levels without affect- ing serum insulin secretion.

The effects of PVEE on serum leptin and adiponectin levels were investigated (Table 1). Leptin exerts a hypophagic effect and is secreted from adipocytes in response to lipid accumulation (13). Serum leptin levels were significantly higher in OVX mice, as compared with shamoperated mice. The PVEE diet attenuated this change, but not significantly (Table 1). Adiponectin decreases blood glucose levels and regulates lipid metabolism (13). Serum adiponectin levels in the OVX-control group were lower than those in the Sham-control group, although the difference was not significant. In addition, there were no significant differences between the levels observed in the control and PVEE-fed groups. Therefore, PVEE improved glucose metabolism without altering leptin or adiponectin levels.

Effects of long-term intake of PVEE on body weight and food intake

Experiment 2 examined the effects of long-term (24wk) intake of a diet containing $5 \mathrm{mg} \cdot \mathrm{kg}^{-1} \cdot \mathrm{d}^{-1}$ PVEE. The body weight of the OVX-control group was significantly greater than that of the Sham-control group (Fig. 3A) and this difference was not affected by the PVEE diet. The final body weights in the Sham-control, Sham-PVEE, OVX-control, and OVX-PVEE groups were $38.7 \pm 0.9, \quad 36.0 \pm 0.2,46.2 \pm 3.6$, and $44.1 \pm 1.9 \mathrm{~g}$, 
A
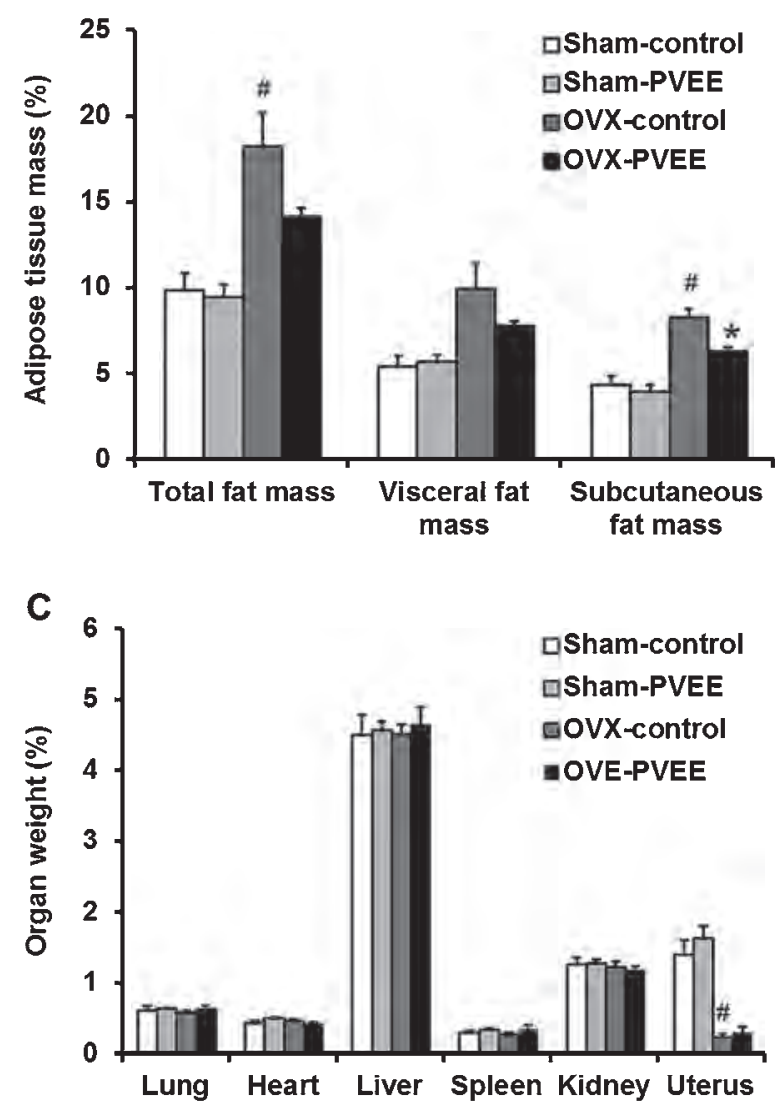

E

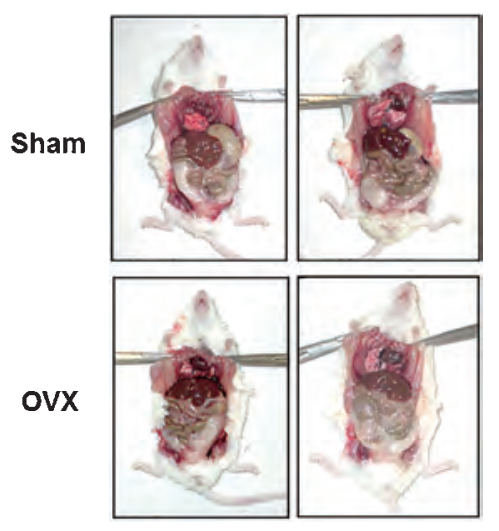

B pink: visceral fat yellow: subcutaneous fat
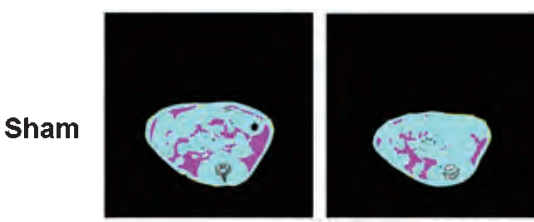

ovx
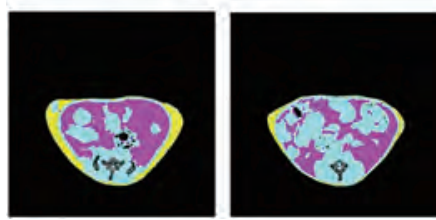

Control

PVEE

D

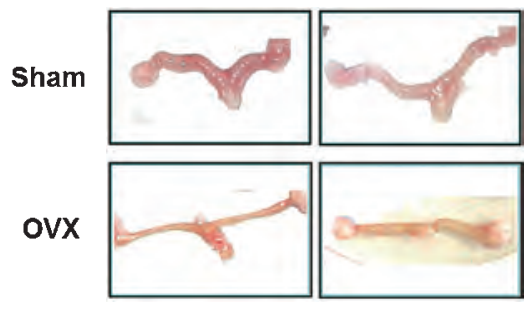

Control

PVEE

PVEE

Fig. 4. Effects of long-term intake of the kudzu vine ethanol extract (PVEE) diet on fat deposits (A, B), organ weights (C), uteri (D), and anatomy (E) at $24 \mathrm{wk}$ in the sham-operated (Sham)-control group ( $n=6)$, Sham-PVEE group $\left(5 \mathrm{mg} \cdot \mathrm{kg}^{-1} \cdot \mathrm{d}^{-1}\right.$, $n=6$ ), ovariectomized (OVX)-control group $(n=6)$, and OVX-PVEE group $\left(5 \mathrm{mg} \cdot \mathrm{kg}^{-1} \cdot \mathrm{d}^{-1}, n=6\right)$. Data are presented as mean \pm standard error. ${ }^{\#} p<0.05$ vs. Sham-control group. ${ }^{*} p<0.05$ vs. OVX-control group.

respectively. During this experiment, food intake did not differ significantly among the study groups (Fig. 3A). The mean food intake in the Sham-control, Sham-PVEE, OVX-control, and OVX-PVEE groups was $3.9 \pm 0.1$, $3.7 \pm 0.1,3.8 \pm 0.1$, and $3.7 \pm 0.1 \mathrm{~g}$, respectively.

Effects of long-term intake of PVEE on fat mass, uterine hypertrophy, organ weight, and anatomy

X-Ray computed tomography analysis showed that the OVX-control group had significantly greater total and subcutaneous fat than the Sham-control group
(Fig. 4A and B). The PVEE diet significantly suppressed the increase in subcutaneous fat in OVX mice. However, there was no significant difference in the level of total and visceral fat between the OVX-control and OVXPVEE groups. The relative total, visceral, and subcutaneous fat values in the OVX-PVEE group were $77.6 \%$, $78.0 \%$, and $76.2 \%$ of the corresponding values in the OVX-control group, respectively.

There were no significant differences in the relative lung, heart, liver, spleen, or kidney weights of the study 
Table 2. Effect of $5 \mathrm{mg} \cdot \mathrm{kg}^{-1} \cdot \mathrm{d}^{-1} \mathrm{kudzu}$ vine ethanol extract (PVEE) diet on serum parameters after $24 \mathrm{wk}$.

\begin{tabular}{lcclc}
\hline & Sham-control & Sham-PVEE & OVX-control & OVX-PVEE \\
\hline Triglyceride (mg/dL) & $99.3 \pm 8.3$ & $103.4 \pm 8.5$ & $135.7 \pm 20.6$ & $79.2 \pm 5.7^{\mathrm{b}}$ \\
Total cholesterol (mg/dL) & $116.2 \pm 14.7$ & $105.3 \pm 14.1$ & $146.6 \pm 3.8$ & $130.7 \pm 4.1$ \\
Glucose (mg/dL) & $200.9 \pm 11.7$ & $211.2 \pm 15.9$ & $260.9 \pm 4.1$ & $188.1 \pm 4.9^{\mathrm{b}}$ \\
\hline
\end{tabular}

Data are presented as mean \pm standard error; ${ }^{b} p<0.05$ vs. OVX-control group. Sham, sham-operated; OVX, ovariectomized.

Table 3. Effect of $20 \mathrm{mg} \cdot \mathrm{kg}^{-1} \cdot \mathrm{d}^{-1}$ puerarin diet on body weight and relative white adipose tissue and uterine weights.

\begin{tabular}{lcccc}
\hline & Sham & OVX-control & OVX-puerarin & OVX-E2 \\
\hline Initial body weight $(\mathrm{g})$ & $30.5 \pm 0.1$ & $31.7 \pm 0.3$ & $31.5 \pm 0.4$ & $31.9 \pm 0.5$ \\
Final body weight at 10 wk (g) & $38.6 \pm 1.0$ & $48.4 \pm 0.8^{\mathrm{a}}$ & $43.6 \pm 0.9^{\mathrm{a}, \mathrm{b}}$ & $41.6 \pm 1.0^{\mathrm{b}}$ \\
White adipose tissue ratio per body weight $(\%)$ & $3.6 \pm 0.4$ & $7.6 \pm 0.8^{\mathrm{a}}$ & $5.4 \pm 0.7$ & $3.3 \pm 0.7^{\mathrm{b}}$ \\
Uterine ratio of body weight (\%) & $0.97 \pm 0.16$ & $0.09 \pm 0.02^{\mathrm{a}}$ & $0.11 \pm 0.01^{\mathrm{a}}$ & $0.18 \pm 0.02^{\mathrm{a}}$ \\
\hline
\end{tabular}

Data are presented as mean \pm standard error; ${ }^{a} p<0.05$ vs. Sham-control group; ${ }^{b} p<0.05$ vs. OVX-control group. Sham, sham-operated; OVX, ovariectomized; E2, $17 \beta$-estradiol.

Table 4. Effect of $20 \mathrm{mg} \cdot \mathrm{kg}^{-1} \cdot \mathrm{d}^{-1}$ puerarin diet on serum parameters after $10 \mathrm{wk}$.

\begin{tabular}{lcccc}
\hline & Sham & OVX-control & OVX-puerarin & OVX-E2 \\
\hline Triglyceride (mg/dL) & $68.3 \pm 10.2$ & $91.4 \pm 9.2$ & $80.5 \pm 6.8$ & $88.7 \pm 11.3$ \\
Total cholesterol (mg/dL) & $170.6 \pm 17.1$ & $195.5 \pm 19.0$ & $188.9 \pm 10.3$ & $195.7 \pm 11.8$ \\
HDL-cholesterol (mg/dL) & $87.3 \pm 8.5$ & $93.2 \pm 9.8$ & $108.5 \pm 4.6$ & $107.0 \pm 6.3$ \\
LDL-cholesterol (mg/dL) & $69.1 \pm 7.2$ & $83.2 \pm 7.1$ & $68.3 \pm 4.3$ & $70.4 \pm 4.7$ \\
Glucose (mg/dL) & $119.7 \pm 5.6$ & $174.8 \pm 9.5^{\mathrm{a}}$ & $121.4 \pm 7.1^{\mathrm{b}}$ & $126.8 \pm 7.3^{\mathrm{b}}$ \\
\hline
\end{tabular}

Data are presented as mean \pm standard error; ${ }^{a} p<0.05$ vs. Sham-control group; ${ }^{b} p<0.05$ vs. OVX-control group. Sham, sham-operated; OVX, ovariectomized; HDL, high-density lipoprotein; LDL, low-density lipoprotein; E2, $17 \beta$-estradiol.

groups (Fig. 4C), suggesting that the PVEE diet suppressed fat accumulation without affecting the weights of other organs. In OVX mice, significant uterine atrophy was observed (Fig. 4C and D) and the PVEE diet did not ameliorate this change. These results were consistent with those of experiment 1 and suggested that the PVEE diet at this dose did not produce any estrogenic activity in vivo. The gross anatomy of the visceral organs in each mouse was examined to check the safety of long-term PVEE administration. No abnormalities were observed (Fig. 4E). Moreover, no abnormal behaviors or adverse effects were observed in Sham-PVEE or OVX-PVEE mice. These results suggested that long-term intake of a diet containing $5 \mathrm{mg} \cdot \mathrm{kg}^{-1} \cdot \mathrm{d}^{-1}$ PVEE safely suppressed the OVX-induced increase in fat mass.

Effects of long-term intake of PVEE on serum parameters

Serum triglyceride, cholesterol, and glucose levels were higher in the OVX-control group than in the Shamcontrol group, although these differences were not significant (Table 2). The PVEE diet significantly attenuated the OVX-associated increase in serum triglyceride and glucose levels. Therefore, long-term intake of a diet containing $5 \mathrm{mg} \cdot \mathrm{kg}^{-1} \cdot \mathrm{d}^{-1}$ PVEE improved serum glucose levels.
Effects of puerarin on body, white adipose tissue, and uterine weight

PVEE is a crude extract that contains many isoflavones and our previous study showed that it contained $\geq 50 \%$ puerarin (8). Therefore, we administered puerarin to OVX mice to investigate whether it had hypoglycemic activity (experiment 3).

The body weight, relative white adipose tissue level, and uterine weights (versus body weight $\times 100$ ) are shown in Table 3. OVX-control mice had significantly increased body weight, increased relative white adipose tissue weight, and decreased relative uterine weights, as compared to the Sham-control mice. Puerarin and E2 treatment significantly reduced the body weight of OVX mice: the final body weights of the OVX-control, OVX-puerarin, and OVX-E2 groups were 48.4 \pm 0.8 , $43.6 \pm 0.9$, and $41.6 \pm 1.0 \mathrm{~g}$, respectively. The OVXinduced increase in the relative white adipose tissue weight tended to be attenuated in the mice treated with the puerarin diet or E2; this effect was statistically significant in E2-treated mice (Table 3). The uterine weight of the OVX-puerarin group was not significantly different from that of the OVX-control group. On the other hand, E2 treatment tended to rescue the uterine atro- 
Table 5. Effect of $20 \mathrm{mg} \cdot \mathrm{kg}^{-1} \cdot \mathrm{d}^{-1}$ puerarin diet on liver lipids after $10 \mathrm{wk}$.

\begin{tabular}{lcccc}
\hline & Sham & OVX-control & OVX-puerarin & OVX-E2 \\
\hline Triglyceride (mg/g liver) & $8.7 \pm 1.4$ & $21.3 \pm 1.6^{\mathrm{a}}$ & $14.6 \pm 1.1^{\mathrm{b}}$ & $16.3 \pm 1.5^{\mathrm{b}}$ \\
Total cholesterol (mg/g liver) & $1.7 \pm 0.2$ & $1.9 \pm 0.2$ & $2.1 \pm 0.2$ & $2.3 \pm 0.2$ \\
\hline
\end{tabular}

Data are presented as mean \pm standard error; ${ }^{a} p<0.01$ vs. Sham-control group; ${ }^{b} p<0.05$ vs. OVX-control group. Sham, sham-operated; OVX, ovariectomized or ovariectomy; E2, $17 \beta$-estradiol.
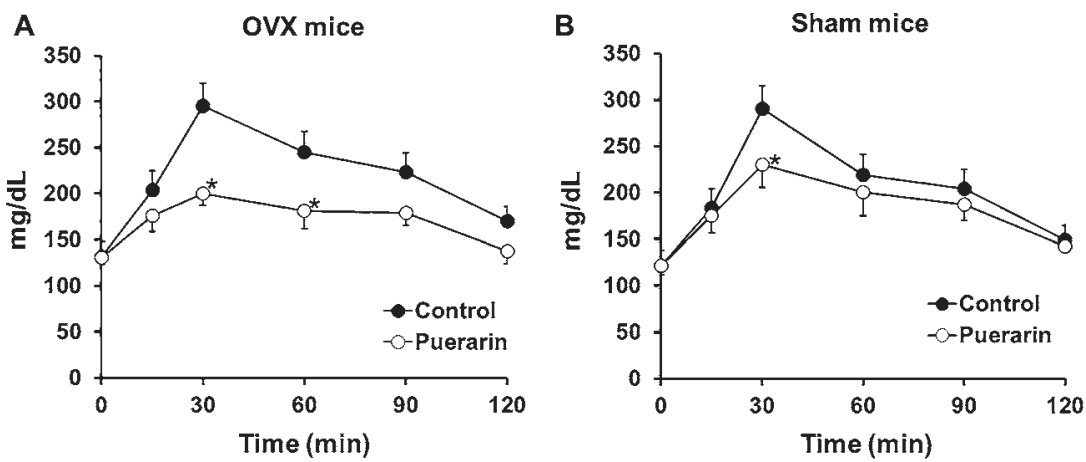

Fig. 5. Effects of puerarin on glucose absorption in ovariectomized (OVX) (A) and Sham-operated (Sham) mice (B). After an overnight fast $(18 \mathrm{~h})$, a glucose solution $\left(1,000 \mathrm{mg} \cdot \mathrm{kg}^{-1}\right)$ with or without puerarin $\left(50 \mathrm{mg} \cdot \mathrm{kg}^{-1}\right)$ was administered orally and blood samples were collected from the tail vein at the indicated time-points. Data are presented as mean \pm standard error. ${ }^{*} p<0.05$ vs. OVX-control group.

phy observed in OVX mice. These results suggested that puerarin ameliorated the OVX-induced increases in body without affecting uterine weight. The effects of puerarin therefore resembled those of PVEE, but not those of E2. Effects of the puerarin diet on serum parameters

The levels of serum triglyceride or total, HDL-, or LDL-cholesterol did not differ between the study groups in this experiment (Table 4). The serum glucose levels were significantly higher in the OVX-control group than in the Sham-control group. Puerarin and E2 attenuated the OVX-associated increase in serum glucose levels. These results suggested that the puerarin diet had hypoglycemic effects in this model that were consistent with those of the PVEE diet.

Effects of the puerarin diet on liver lipids

Analysis of liver triglyceride and total cholesterol levels indicated that OVX-control mice had significantly higher liver triglyceride levels than the Sham-control mice (Table 5). Treatment with puerarin or E2 attenuated these increases. In contrast, the study groups showed no differences in the total liver cholesterol levels. These results suggested that puerarin ameliorated the OVX-induced increase in liver triglyceride levels, without altering cholesterol metabolism.

Effects of puerarin on glucose absorption

To explore whether puerarin suppressed glucose absorption in OVX mice, oral glucose tolerance tests were performed (experiment 4). As shown in Fig. 5A, the maximum serum glucose level observed $30 \mathrm{~min}$ after glucose administration to OVX-control mice was $295.4 \pm 24.3 \mathrm{mg} / \mathrm{dL}$. The corresponding level in the OVX-puerarin group was $199.7 \pm 12.9 \mathrm{mg} / \mathrm{dL}$. Puerarin also significantly decreased serum glucose level observed 30 min after glucose administration in Sham mice (Fig. 5B). Thus, puerarin improved glucose tolerance in OVX and Sham mice. These results suggested that the hypoglycemic effect of puerarin may reflect its suppression of intestinal glucose absorption.

\section{DISCUSSION}

We used OVX mice as a model of postmenopausal diabetes. Decreases in female hormone secretion increase insulin resistance $(4,5)$. OVX mice showed significantly increased weight gain, adipose tissue mass, and serum glucose levels, as compared to sham-operated mice, as described previously $(9,14,15)$. Dietary PVEE decreased this elevation in the serum glucose level without significantly affecting serum insulin levels (Table 1). The body weights (Fig. 1A) and relative white adipose tissue weights (Fig. 2) in the OVX mice treated with PVEE for 8 wk were lower than those in the OVX-control mice. These results indicated that the PVEE diet suppressed the OVX-induced increases in body weight and fat mass by improving glucose metabolism.

OVX (with or without E2) did not increase serum cholesterol or triglyceride levels in this study (Tables 1-4). Stubbins et al. showed that in OVX mice, a low-fat diet did not alter serum triglyceride levels, while a high-fat diet increased serum triglyceride levels (16). In addition, the authors reported no significant difference between OVX-control and E2-injected OVX mice. Hence, the present results were partially in agreement with those of Stubbins et al. (16), because a normal diet was used in the present study. 
Some reports suggest that OVX treatment increases food intake in female rats $(17,18)$. However, in this study, there were no significant changes in the food intake between the OVX group and Sham group. The difference between the results of this study and that of other reports may be due to animal strain and age.

In experiment 2, the effect of long-term intake of a lower amount of PVEE $\left(5 \mathrm{mg} \cdot \mathrm{kg}^{-1} \cdot \mathrm{day}^{-1}\right.$, approximately $0.17 \mathrm{mg}$ PVEE isoflavone $\cdot \mathrm{d}^{-1} \cdot$ animal $^{-1}$ ) was examined. This dosage was below the recommended daily dietary isoflavonoid allowance in Japan (15$22 \mathrm{mg}$ supplementation $\cdot \mathrm{d}^{-1}$ for a $60-\mathrm{kg}$ man), which corresponded to $0.25-0.37 \mathrm{mg} \cdot \mathrm{d}^{-1}$. animal $^{-1}$. Longterm intake of PVEE ameliorated OVX-induced increases in subcutaneous fat mass and serum glucose levels, without causing any abnormalities in body weight, food intake, uterine and other organ weights, or gross anatomy of the visceral organs. These results suggested that long-term intake of the PVEE diet at this dose safely reduced hyperglycemia and fat accumulation.

Puerarin was the most probable candidate for the active species present in PVEE, since it was the most abundant isoflavone $(8)$. The present study found that ingestion of a puerarin diet ameliorated the OVXinduced elevations of serum glucose levels, body weight, and white adipose tissue weight in a similar manner to the PVEE diet (Tables 3 and 4). These data were consistent with those of a recent study showing that puerarin (100 and $200 \mathrm{mg} \cdot \mathrm{kg}^{-1}$ body weight) suppressed weight gain in rats fed a high-fat diet (19). Therefore, the glucose metabolism-improving actions of PVEE were most likely due to puerarin, rather than other minor isoflavones, although further studies for the effective dosage might be needed.

Puerarin (daidzein-8-C-glycoside) may be hydrolyzed in vivo to produce daidzein and equol, which may also contribute to the effects of PVEE. However, this appears unlikely since the $C$-glycoside bond in puerarin is very resistant to mouse microflora (20). The observation by Prasain et al. (21) that non-metabolized puerarin and a small amount of its glucuronide were predominantly absorbed and detected at the early stage of feeding in the serum of puerarin-fed rats is also consistent with our preliminary data (data not shown). Furthermore, puerarin was shown to be widely distributed in tissues (kidney, liver, lung, pancreas, heart, eye, and brain) of puerarin-treated rats (21-23). Hence, most dietary puerarin may be absorbed into the systemic circulation without deglycosylation or conjugation.

E2 treatment protected against OVX-induced increases in adipocyte diameter and insulin resistance in female mice fed a high-fat diet (16). There are some reports that isoflavones act as estrogen mimetics (24, 25 ). The uterine atrophy induced by estrogen deficiency was unaltered by dietary PVEE or puerarin, whereas injection of E2 prevented this loss of uterine weight in OVX mice (Fig. 1, Fig. 4, Table 3). Thus, PVEE and puerarin appeared to behave in a different manner to E2. We previously reported that puerarin did not bind to estrogen receptor $\alpha$ or $\beta$, while daidzein can bind to estrogen receptors (26). Therefore, the mechanism by which puerarin improved glucose metabolism probably does not involve estrogen-like activity.

Intravenous injection of puerarin $\left(15 \mathrm{mg} \cdot \mathrm{kg}^{-1}\right.$ body weight) in streptozotocin-induced diabetic rats was reported to decrease plasma glucose levels by increasing glucose transporter 4 expression levels in the soleus muscle (27). Although this may explain the hypoglycemic effects observed, PVEE did not change glucose transporter 4 expression levels in the soleus muscle in the present study (data not shown). These different results may be due to differences in the mouse strain studied, or the administration dose or route.

To examine other potential mechanisms by which puerarin improved glucose metabolism, oral glucose tolerance tests were performed. As shown in Fig. 5, oral administration of puerarin improved glucose tolerance in OVX and Sham mice. These results were consistent with the report by Meezan et al. indicating that both oral and intraperitoneal administration of puerarin suppressed glucose absorption in a mouse model of type 2 diabetes mellitus (28). Therefore, the suppression of intestinal glucose absorption is likely involved in the hypoglycemic effects observed in the present study. The liver triglyceride levels were lower in the OVX-puerarin group than in the OVX-control group (Table 5). High glucose conditions stimulated expression of genes involved in fatty acid synthesis in human hepatoma cell line (HepG2 cells) (29) and fatty acids liberated were accumulated as triglyceride in liver $(30,31)$. Therefore, liver triglyceride synthesis might decrease as a result of the reduced glucose absorption.

In conclusion, the present study demonstrated that dietary PVEE attenuated the elevation of body weight, adipose tissue mass, and serum glucose levels observed in OVX mice. However, further studies are needed to clarify the molecular mechanism underlying these observations. The experimental diets were not associated with any abnormalities in the mice tested in the present study, indicating that PVEE and puerarin may provide useful dietary supplements for ameliorating postmenopausal diabetes.

\section{Acknowledgments}

This study was partly funded and supported by the Local Collective Research and Development Program of Nara (2006-2010) from the Japan Science and Technology Agency (JST). This work was also supported by the Strategic Project to Support the Formation of Research Bases at Private Universities: Matching Fund Subsidy from MEXT (Ministry of Education, Culture, Sports, Science and Technology), 2011-2015 (S1101035).

\section{REFERENCES}

1) Gaspard U. 2009. Hyperinsulinaemia, a key factor of the metabolic syndrome in postmenopausal women. Maturitas 62: 362-365.

2) Lima R, Wofford M, Reckelhoff JF. 2012. Hypertension in postmenopausal women. Curr Hypertens Rep 14: 254-260. 
3) Formiguera X, Canton A. 2004. Obesity: epidemiology and clinical aspects. Best Pract Res Clin Gastroenterol 18: 1125-1146.

4) Saengsirisuwan V, Pongseeda S, Prasannarong M, Vichaiwong K, Toskulkao C. 2009. Modulation of insulin resistance in ovariectomized rats by endurance exercise training and estrogen replacement. Metabolism 58: $38-47$.

5) Lemieux C, Picard F, Labrie F, Richard D, Deshaies Y. 2003. The estrogen antagonist EM-652 and dehydroepiandrosterone prevent diet- and ovariectomy-induced obesity. Obes Res 11: 477-490.

6) Prasain JK, Peng N, Rajbhandari R, Wyss JM. 2012. The Chinese Pueraria root extract (Pueraria lobata) ameliorates impaired glucose and lipid metabolism in obese mice. Phytomedicine 20: 17-23.

7) Peng N, Prasain JK, Dai Y, Moore R, Arabshahi A, Barnes S, Carlson S, Wyss JM. 2009. Chronic dietary kudzu isoflavones improve components of metabolic syndrome in stroke-prone spontaneously hypertensive rats. J Agric Food Chem 57: 7268-7273.

8) Tanaka T, Tang H, Yu F, Michihara S, Uzawa Y, Zaima N, Moriyama T, Kawamura Y. 2011. Kudzu (Pueraria lobata) vine ethanol extracts improve ovariectomyinduced bone loss in female mice. J Agric Food Chem 59: 13230-13237.

9) Choi JS, Song J. 2009. Effect of genistein on insulin resistance, renal lipid metabolism, and antioxidative activities in ovariectomized rats. Nutrition 25: 676-685.

10) Yonezawa R, Wada T, Matsumoto N, Morita M, Sawakawa K, Ishii Y, Sasahara M, Tsuneki H, Saito S, Sasaoka T. 2012. Central versus peripheral impact of estradiol on the impaired glucose metabolism in ovariectomized mice on a high-fat diet. Am J Physiol Endocrinol Metab 303: 445-456.

11) Feigh M, Andreassen KV, Hjuler ST, Nielsen RH, Christiansen C, Henriksen K, Karsdal MA. 2013. Oral salmon calcitonin protects against impaired fasting glycemia, glucose intolerance, and obesity induced by high-fat diet and ovariectomy in rats. Menopause 20: 785-794.

12) Bligh EG, Dyer WJ. 1959. A rapid method of total lipid extraction and purification. Can J Biochem Physiol 37: 911-917.

13) Nishiumi S, Bessyo H, Kubo M, Aoki Y, Tanaka A, Yoshida K, Ashida H. 2010. Green and black tea suppress hyperglycemia and insulin resistance by retaining the expression of glucose transporter 4 in muscle of high-fat diet-fed C57BL/6J mice. J Agric Food Chem 58: 12916-12923.

14) Richard D. 1986. Effects of ovarian hormones on energy balance and brown adipose tissue thermogenesis. Am J Physiol 250: 245-249.

15) Na XL, Ezaki J, Sugiyama F, Cui HB, Ishimi Y. 2008. Isoflavone regulates lipid metabolism via expression of related genes in OVX rats fed on a high-fat diet. Biomed Environ Sci 21: 357-364.

16) Stubbins RE, Najjar K, Holcomb VB, Hong J, Núñez NP.
2012. Oestrogen alters adipocyte biology and protects female mice from adipocyte inflammation and insulin resistance. Diabetes Obes Metab 14: 58-66.

17) Jankowiak R, Stern JJ. 1974. Food intake and body weight modifications following medial hypothalamic hormone implants in female rats. Physiol Behav 12: 875-879.

18) Chen Y, Heiman ML. 2001. Increased weight gain after ovariectomy is not a consequence of leptin resistance. Am J Physiol Endocrinol Metab 280: 315-322.

19) Zhang W, Liu CQ, Wang PW, Sun SY, Su WJ, Zhang HJ, Li XJ, Yang SY. 2010. Puerarin improves insulin resistance and modulates adipokine expression in rats fed a highfat diet. Eur J Pharmacol 649: 398-402.

20) Kim DH, Yu KU, Bae EA, Han MJ. 1998. Metabolism of puerarin and daidzin by human intestinal bacteria and their relation to in vitro cytotoxicity. Biol Pharm Bull 21: 628-630.

21) Prasain JK, Jones K, Brissie N, Moore R, Wyss JM, Barnes S. 2004. Identification of puerarin and its metabolites in rats by liquid chromatography-tandem mass spectrometry. J Agric Food Chem 52: 3708-3712.

22) Prasain JK, Peng N, Moore R, Arabshahi A, Barnes S, Wyss JM. 2009. Tissue distribution of puerarin and its conjugated metabolites in rats assessed by liquid chromatography-tandem mass spectrometry. Phytomedicine 16: $65-71$.

23) Barnes S, Prasain J, D'Alessandro T, Arabshahi A, Botting N, Lila MA, Jackson G, Janle EM, Weaver CM. 2011. The metabolism and analysis of isoflavones and other dietary polyphenols in foods and biological systems. Food Funct 2: 235-244.

24) Choi SY, Ha TY, Ahn JY, Kim SR, Kang KS, Hwang IK, Kim S. 2008. Estrogenic activities of isoflavones and flavones and their structure-activity relationships. Planta Med 74: 25-32.

25) Vitale DC, Piazza C, Melilli B, Drago F, Salomone S. 2013. Isoflavones: estrogenic activity, biological effect and bioavailability. Eur J Drug Metab Pharmacokinet 38: 15-25.

26) Michihara S, Tanaka T, Uzawa Y, Moriyama T, Kawamura Y. 2012. Puerarin exerted anti-osteoporotic action independent of estrogen receptor-mediated pathway. J Nutr Sci Vitaminol 58: 202-209.

27) Hsu FL, Liu IM, Kuo DH, Chen WC, Su HC, Cheng JT. 2003. Antihyperglycemic effect of puerarin in streptozotocin-induced diabetic rats. J Nat Prod 66: 788-792.

28) Meezan E, Meezan EM, Jones K, Moore R, Barnes S, Prasain JK. 2005. Contrasting effects of puerarin and daidzin on glucose homeostasis in mice. J Agric Food Chem 53: $8760-8767$.

29) Mitro N, Mak PA, Vargas L, Godio C, Hampton E, Molteni V, Kreusch A, Saez E. 2007. The nuclear receptor LXR is a glucose sensor. Nature 445: 219-223.

30) Gibbons GF. 1990. Assembly and secretion of hepatic very-low-density lipoprotein. Biochem J 268: 1-13.

31) Gibbons GF, Islam K, Pease RJ. 2000. Mobilisation of triacylglycerol stores. Biochim Biophys Acta 1483: 37-57. 